\title{
'In-Situ Split' Liver Resection/ALPPS - Historical Development and Current Practice
}

\author{
Hans J. Schlitt ${ }^{a} \quad$ Christina Hackl $^{a}$ Sven Arke Lang ${ }^{a, b}$ \\ a Department of Surgery, University of Regensburg Medical Center, Regensburg, Germany; \\ ${ }^{b}$ Department of Surgery, University of Freiburg, Freiburg i.Br., Germany
}

\section{Keywords}

Liver resection - Technique - In-situ split - ALPPS . Indication

\section{Summary}

Background: Liver tumors that are extensive, multifocal, or critically located frequently require advanced techniques of liver resection including 'functional augmentation' of liver segments. Here we describe the invention and stepwise establishment of a new technique - termed 'in-situ split' liver resection or 'ALPPS' - enabling liver resection in certain situations. Methods: The development of the technique in the first and the subsequent 8 patients in the index center, and also the method's spread throughout Germany and the world were reviewed. Results: In 2007, in the first patient, the new technique was developed intraoperatively by necessity. Due to the convincing outcome, it was deliberately applied again several months later in another patient, and thereafter (sparsely) used for liver resection for various indications. Following oral communication, the method spread throughout Germany, and later - mainly following the publication of the initial multicentric German series - very quickly disseminated worldwide. Currently, it is used for a very (if not overly) broad spectrum of indications by many hepatobiliary surgery centers. Conclusion: In-situ split/ALPPS is a newly developed technique for liver resection, which was established for very specific situations. This method has created a hype, and is currently used rather generously by many centers worldwide.

(C) 2017 S. Karger GmbH, Freiburg

\section{Introduction}

The limiting factor for (potentially curative) liver resection for certain primary or secondary tumors is the amount of functionally sufficient liver tissue that must remain [1]. If too small a left lateral section were to remain, preoperative embolization of the right portal vein, with or without embolization of the portal vein branches to liver segment IV, is an established technique [2]. However, the volume increase in the left lateral section is between 20 and $70 \%$ within approximately 6 weeks, i.e. highly variable and taking a rather long time $[3,4]$. Moreover, in certain tumors, e.g. hilar cholangiocarcinoma (hCCC; Klatskin tumor), the preoperative embolization of the right side does no longer allow for an intraoperative strategy change to a left hepatectomy if required. Therefore, this approach is suboptimal in several situations. Moreover, it does not allow highly sensitive imaging of the left lateral section for the detection of small additional tumor load by intraoperative ultrasound (or even contrast-enhanced ultrasound) prior to deciding on the treatment strategy. Thus, a novel strategy was required to overcome these issues. The new technique, the development of which is described in this paper, is based on technical experience with both liver resection and in-situ split liver transplantation. This technique, termed 'in-situ split' liver resection and later found in the literature as ALPPS (associating liver partition and portal vein ligation for staged hepatectomy), was developed by the author by chance, and then started to conquer the hepato-pancreato-biliary (HPB) surgery world, first in Germany and then throughout the world. Here we describe the history of this development including details of the first case.

\section{Patients}

The indications, perioperative considerations, as well as perioperative data of our first 9 patients were analyzed retrospectively. These patients underwent surgery between September 2007 and 2010. Details of the patients and procedures are given below. 
Fig. 1. First patient (hilar cholangiocarcinoma, Klatskin tumor) in whom an 'in-situ split' liver resection/ ALPPS was performed in September 2007.

a Contrast-enhanced computed tomography (CT) scan prior to the first surgery: dilated bile ducts, particularly in the left lateral section of the liver, in spite of preoperative drainage by endostent + percutaneous transhepatic cholangiography drainage (PTCD). b Contrastenhanced CT scan 8 days after the first surgery with marked volume increase in the left lateral section. c Contrast-enhanced CT scan 7 day after the second surgery showing marked size increase of the remaining left lateral section.

\section{Methods}

In addition to the analysis of our patients, a non-systematic review of the literature as well as of conference reports and personal communications was performed to form the basis for the further assessment of the use of the novel technique.

\section{First Case of 'In-Situ Split' Liver Resection}

A 49-year-old female patient presented with painless jaundice from suspected hCCC (Klatskin tumor) Bismuth type IV. Because of high serum bilirubin $(15 \mathrm{mg} / \mathrm{dl})$ with dilated bile ducts on both sides of the liver, a biliary stent was initially placed endoscopically to the right liver in a peripheral hospital, and the patient was then referred to our center. Upon admission, serum bilirubin was $5 \mathrm{mg} /$ dl. After a review of all imaging results, it was decided to perform a right trisectionectomy after preoperative decompression of the left lateral ducts (fig. 1a). This could not be achieved via endoscopic retrograde cholangiography (ERC); therefore, a percutaneous transhepatic cholangiography and drainage (PTCD) was placed via the right side into the left ductal system. Bilirubin was $2 \mathrm{mg} / \mathrm{dl}$, and surgery was performed on September 3, 2007. A preoperative volumetry had not been performed, and during surgery, the left lateral section was found to be too small (fig. 1a): retrospective volumetry showed $370 \mathrm{~g}$ with a patient body weight of $78 \mathrm{~kg}$. Hence it was spontaneously decided during surgery to try and quickly induce hypertrophy of the left lateral section by de-portalizing the right liver while already performing the parenchymal dissection along the right side of the falciform ligament, thereby completely devascularizing segment IV in preparation for the final resection to be performed in a second step. Moreover, the left bile duct was divided at the base of the round ligament, about $2 \mathrm{~cm}$ away from the hepatic bifurcation, and a frozen section of the proximal bile duct resection margin was shown to be tumor-free. The bile duct towards the bifurcation was then suture-closed, and a Roux-en-Y hepaticojejunostomy was performed to the left duct. In order to facilitate the completion surgery, the right hepatic artery was dissected on the right side of the hilum and encircled with a vessel loop. Moreover, the right liver, including segment I, was mobilized from the retroperitoneum and from the vena cava. Finally, the right and middle hepatic veins were visualized; the middle hepatic vein was divided and the right hepatic vein was encircled with a vessel loop. Following placement of several drains, the abdomen was closed.

The patient recovered quickly from surgery; liver function was normal, and a computed tomography (CT) scan performed after 8 days (fig. 1b) astonishingly showed that the left lateral section had obviously increased to $723 \mathrm{ml}$, i.e. by more than $90 \%$. Therefore, it was decided to perform completion surgery the following day. During this second operation, the distal bile duct, the right hepatic artery, and the right hepatic vein were divided, and the extended right liver including segment I was removed. Then, a systematic lymphadenectomy was performed prior to closure of the abdomen. The patient recovered quickly from surgery (fig. 1c) and could be discharged 10 days later.

Histology of the resected specimens showed a Klatskin tumor (hCCC) with 2 lymph node metastases as well as microscopic peritoneal carcinomatosis in the lesser omentum (pT3, pN1(2/8), $\mathrm{pM} 1_{\text {per }}$, local R0). As already suspected from this histology, the patient started to develop ascites and signs of overt peritoneal carcinomatosis about 6 months after surgery and died another 8 months later from tumor recurrence.

\section{Subsequent Patients Undergoing 'In-Situ Split' Liver Resection in Index Center}

Although the technique had worked well and had led to very rapid hypertrophy of a rather small left lateral section, it was not applied again until more than half a year later (June 2008). At this time, it was used in a rather complex patient with rectal cancer and multiple synchronous liver metastases. After systemic chemotherapy, the first operation consisted of an abdominoperineal rectum resection with terminal descendostomy and 'in-situ splitting' of the liver. Liver segments II and III proved to be tumor-free on intraoperative ultrasound, while all other segments, including segment I, had tumor involvement. This time, the right portal vein was divided and the right hepatic artery marked with a vessel loop on the 
right side of the hilum, the right liver including segment I was mobilized from the vena cava, and the right hepatic vein was encircled with a vessel loop. Then the liver parenchyma was completely divided to the right of the falciform and round ligaments with the resection line then turning left in front of segment I. Finally, the middle hepatic vein was encircled with a vessel loop, and after placement of drains the operation was completed. Again, there was a rapid increase in the CT-measured volume of segments II and III from $211 \mathrm{ml}$ prior to the first operation to $373 \mathrm{ml}$ after 9 days (i.e. $76 \%$ volume increase) (fig. 2) so that the second surgery could be performed on day 10 with an uncomplicated postoperative course. Another patient, this time with metachronous colorectal liver metastases, underwent a similar procedure in September 2008. In this patient, an injury to the left bile duct occurred during the second surgery which was addressed by an end-to-end reconstruction of the duct. However, after a few days, biliary leakage required reoperation, which revealed necrosis of the duct at the anastomosis site so that a hepaticojejunostomy had to be performed. Again, after several days, leakage was noticed again with erosion bleeding from the hepatic artery, again necessitating emergency surgery. The patient finally died from multiorgan failure a few days later.

In 2009, the new technique was applied in 2 patients both suffering from intrahepatic cholangiocellular carcinoma (iCCC) (fig. 3a). In both patients, the 'standard' procedure was performed with complete parenchymal division to the right of the falciform and round ligaments, with a small part of subsegment IVa preserved in 1 of the 2 patients. These were the only 2 patients where the increase in volume of the left lateral section was below 50\%, and in 1 patient the remnant liver volume prior to the second step of surgery even remained below $0.5 \%$ of the body weight; this patient had already undergone prior interventional embolization of the right portal vein without relevant hypertrophy of the left lateral section (fig. 3b). Nevertheless, the second step of surgery was performed (fig. 3c), and both patients recovered well.

In 2010, a total of 4 'in-situ split' liver resections were performed; 3 patients had colorectal metastases, and 1 patient suffered from metachronous liver metastases from cervical cancer.

In summary, all patients underwent extended right hepatectomy (trisectionectomy). Segment I had to be removed in about half of the patients and could be spared in the other half.

\section{Application of the Technique in Other Centers in Germany}

Since the first case, the new technique was initially only informally communicated by the author and some of the senior surgeons of the department on a national level. It was then rapidly taken up and applied by a number of colleagues in Germany, particularly in Göttingen (Aiman Obed), Mainz (Hauke Lang), Tübingen (Silvio Nadalin and Alfred Königsrainer), Giessen (Winfried Padberg), and Hamburg (Karl Oldhafer). In order to accumulate an appropriate number of patients for the first report on this method, 5 German centers joined their experience with a total of

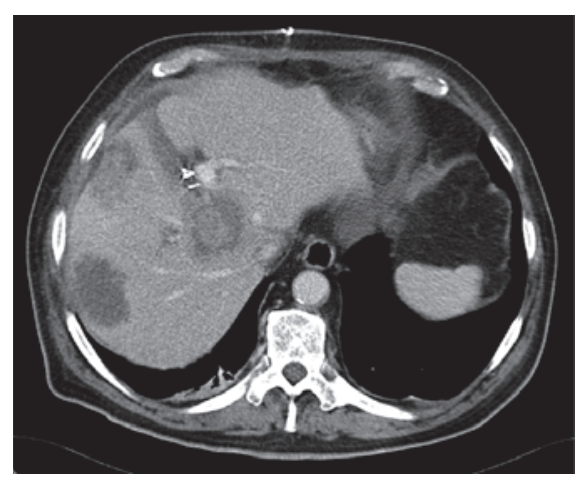

25 patients for analysis in 2011, which led to the sentinel publication of 'in-situ split' liver resection in Annals of Surgery in 2012 [5]. Since then, many German centers have embraced the new method.

\section{Application of the Technique Worldwide}

The new technique appeared on the international podium when Hauke Lang presented 3 cases as a poster at the 9th E-AHPBA (European-African Hepato-Pancreato-Biliary Association) meeting in Cape Town, South Africa, in April 2011 [6], where it was viewed rather controversially. After this presentation, Eduardo de Santibañes from Buenos Aires was one of the first to pick up the idea and start using the concept outside of Europe. After the formal publication of the German cases in Annals of Surgery in March 2012 [5], the technique became known worldwide. An accompanying editorial of our paper by the journal editor Pierre-Alain Clavien together with Eduardo de Santibañes introduced the acronym 'ALPPS' for our 'in-situ split' liver resection [7]. In fact, the paper created enormous publicity for this method which then underwent various modifications by many groups worldwide, such as 'miniALPPS' [8], 'partial ALPPS’ [9], and 'laparoscopic ALPPS' [10, 11]. In fact, more than 3,000 publications on this topic have appeared on PubMed since our initial description, and it has been the single focus of several conferences [12]. Moreover, an ALPPS registry was initiated, and already quite a number of publications have been generated from it and about 1,000 cases have been documented in it over the last 5 years $[13,14]$. Most data in the registry come from centers in Europe (mainly Switzerland, Germany, France, Spain, UK, Belgium, Sweden) and from South America (Argentina, Brazil). Also, when looking at the published literature, it appears that the technique is now broadly used throughout the world, with the exception of the USA where only few surgeons seem to have embarked on it.

It is interesting that in some centers, $10-20 \%$ of all liver resection appear to be ALPPS resections. In our tertiary referral center, where about 200 mainly major liver resections are performed annually, we only come across about 4 cases per year where this approach is deemed helpful and necessary, representing about $2 \%$ of our caseload. In fact, in many cases of 'in-situ split'/ALPPS resection presented in reports and at conferences or documented in the 
Fig. 3. Patient with intrahepatic cholangiocellular carcinoma (iCCC), after prior portal vein embolization (PVE), undergoing 'in-situ split' liver resection/ALPPS in 2009. a Contrast-enhanced computed tomography (CT) scan prior to the first surgery, post PVE; body weight $78 \mathrm{~kg}$; left lateral section of the liver: $190 \mathrm{ml}(0.24 \%$ of body weight). b Contrast-enhanced CT scan prior to first surgery, post PVE; embolization material in the right
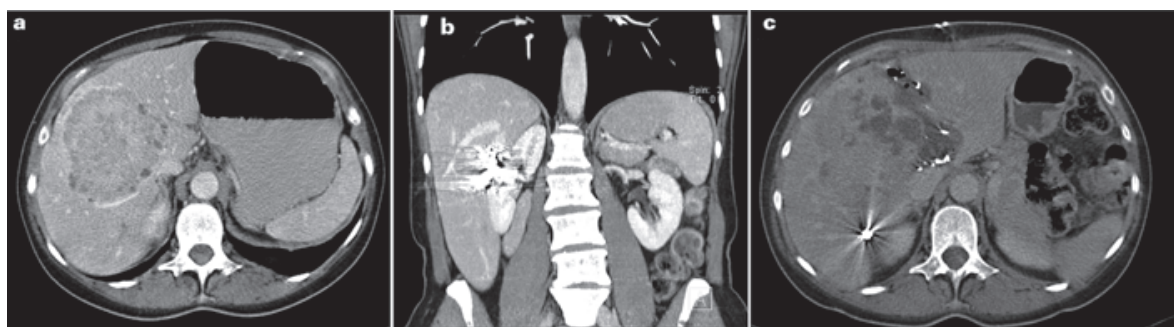
liver. c Contrast-enhanced CT scan prior to the second surgery after 9 days; body weight $78 \mathrm{~kg}$; left lateral section: $275 \mathrm{ml}$ ( $0.35 \%$ of body weight).

Fig. 4. Patient not suitable for 'in-situ split' liver resection/ALPPS but for 'standard' 2-stage hepatectomy (TSH), with synchronous colorectal liver metastases after neoadjuvant/ conversion chemotherapy.

a Contrast-enhanced computed tomography (CT) scan showing bilateral large colorectal liver metastases prior to chemotherapy.

b Contrast-enhanced CT scan after 4 months of chemotherapy showing excellent response of the liver metastases, prior to the first surgery. c Intraoperative view at the end of the first surgery after resection of 5 metastases from the left (including segment IV) and the right portal vein division, in preparation for later removal of metastases on the right side by right hepatectomy. Preoperatively, 'in-situ
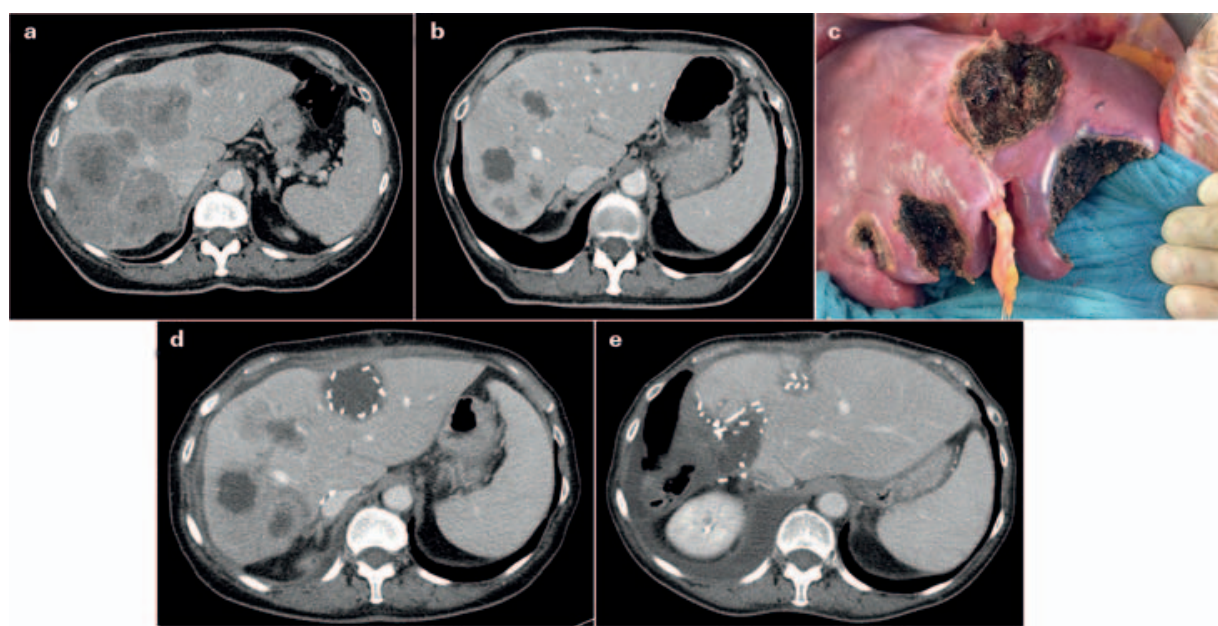
split' liver resection/ALPPS had been planned;

due to a larger than expected number of metastases in the left lobe (detected by intraoperative contrast-enhanced ultrasound), the strategy was changed intraoperatively. d Contrast-enhanced CT scan 8 weeks after primary surgery showing increase in volume of the left liver (including segments IV and I), prior to the second surgery following TSH/portal vein ligation. e Contrast-enhanced CT scan 5 weeks after the second surgery (right hepatectomy with minor parts of segment IVa).

registry, to our mind, this approach is neither necessary nor useful [13]. An example of such a patient with extensive colorectal liver metastases is demonstrated in figure 4 .

\section{Discussion}

The first case in which an 'in-situ split' liver resection was performed occurred spontaneously, i.e. without prior planning. The concept was developed intraoperatively to make an otherwise nonresectable tumor resectable, in a novel 2-stage procedure. This new procedure was based on extensive experience with complex liver resection and standard methods of parenchymal augmentation such as portal vein embolization (PVE) and 2-stage hepatectomy (TSH), as well as experience from the (mainly pediatric) field of split liver transplantation, namely 'in-situ splitting'. The approach was therefore termed 'in-situ split' liver resection. The spontaneous development of this technique demonstrates that in complex situations surgeons should try to find new solutions based on experiences even from a different surgical field. It also shows that new surgical strategies cannot always be designed by thoughtful planning and introduced systematically, but that spontaneous ideas can also be very fruitful.
Interestingly, the indication for the novel procedure in the first patient turned out to be rather suboptimal, and it has rarely been used for this indication since (including our center) [15-17]. However, a broad range of other indications in which the technique is useful have emerged and include many primary (predominantly hepatocellular carcinoma and iCCC) [18] as well as secondary liver tumors, i.e. metastases. In fact, as shown by the ALPPS registry data, colorectal liver metastases currently represent the primary indication for 'in-situ split' liver resection/ALPPS in our center and worldwide [14, 15, 19-22].

It has to be kept in mind that 'in-situ split' liver resection/ ALPPS is not a completely novel technique standing on its own, but that it is rather an additional new tool in the surgical armament for the treatment of liver tumors. Compared to other available techniques, it has some advantages and some disadvantages, which have to be considered in the decision-making process in each individual case: i) interventional PVE (or surgical portal vein ligation) if hypertrophy of the complete left liver (including segment IV) is to be achieved; ii) TSH if volume increase is not specifically dependent on the left lateral section; iii) combination of resection and local ablation (either simultaneously or sequentially); and iv) 1-stage surgery, even when the volume of the remnant liver is marginally low. Thus, a comparison of the various techniques [22] is 
generally not very informative since all have slightly different indications. As mentioned above, in our tertiary referral center with about 200 (mostly major) liver resections per year, with the differential use of all the techniques, 'in-situ split' liver resection/ALPPS is performed in only about $2 \%$, i.e. approximately 4 cases, per year.

Considering the literature on 'in-situ split' liver resection/ ALPPS over the last 5 years, as well as listening to oral presentations about the topic, it emerges that many centers/surgeons use the technique for a much broader range of indications. These include cases of anatomic right hepatectomy as well as trisectionectomy in patients with an appropriately large remnant left lateral section, with or without segment I. It appears that many centers may use the method more frequently because it is currently 'fashionable', or because it is surgically and scientifically (publications) interesting, or in order to increase the competitiveness of the respective center (marketing). However, a highly sensible and selective use of the technique is essential in order to achieve the highest benefit and the lowest risk for each individual patient.
In conclusion, we are astonished at the publicity the in-situ split' liver resection/ALPPS procedure has gained; it has almost become a 'fashion'. We consider it less of a 'great new technique' but rather a new addition to the armament of highly experienced HPB surgeons. Every surgeon using this technique should have the entire spectrum of techniques and interventions available in order to select the optimal surgical approach for each individual patient. Sensible selection of patients who may benefit from the use of 'insitu split' liver resection/ALPPS - with or without some of the modifications described in the recent literature or that may be developed in the future - is essential, as is the continued extensive (and truthful) discussion and reporting of results. Clearly no technique can overrule tumor biology [21].

\section{Disclosure Statement}

There is no conflict of interest by any of the authors.

\section{References}

1 Guglielmi A, Ruzzenente A, Conci S, Valdegamberi A, Iacono C: How much remnant is enough in liver resection? Dig Surg 2012;29:6-17.

2 Ironside N, Bell R, Bartlett A, McCall J, Powell J, Pandanaboyana S: Systematic review of perioperative and survival outcomes of liver resections with and without preoperative portal vein embolization for colorectal metastases. HPB (Oxford) 2017;19:559-566.

$\checkmark 3$ Pandanaboyana S, Bell R, Hidalgo E, Toogood G, Prasad KR, Bartlett A, Lodge JP: A systematic review and meta-analysis of portal vein ligation versus portal vein embolization for elective liver resection. Surgery 2015;157:690-698.

4 Shindoh J, Vauthey JN, Zimmitti G, Curley SA, Huang SY, Mahvash A, Gupta S, Wallace MJ, Aloia TA: Analysis of the efficacy of portal vein embolization for patients with extensive liver malignancy and very low future liver remnant volume, including a comparison with the associating liver partition with portal vein ligation for staged hepatectomy approach. J Am Coll Surg 2013;217:126-133.

5 Schnitzbauer AA, Lang SA, Goessmann H, Nadalin S, Baumgart J, Farkas SA, Fichtner-Feigl S, Lorf T, Goralcyk A, Hörbelt R, Kroemer A, Loss M, Rümmele P, Scherer MN, Padberg W, Königsrainer A, Lang H, Obed A, Schlitt HJ: Right portal vein ligation combined with in situ splitting induces rapid left lateral liver lobe hypertrophy enabling 2-staged extended right hepatic resection in small-for-size settings. Ann Surg 2012;255:405-414.

6 Baumgart J, Lang S, Lang H: A new method for induction of liver hypertrophy prior to right trisectionectomy: a report of three cases. HPB (Oxford) 2011;13: $71-72$.

7 De Santibanes E, Clavien PA: Playing Play-doh to prevent postoperative liver failure: the 'ALPPS' approach. Ann Surg 2012;255:415-417.
De Santibanes E, Alvarez FA, Ardiles V, Pekolj J, de Santibanes M: Inverting the ALPPS paradigm by minimizing first stage impact: the mini-ALPPS technique. Langenbecks Arch Surg 2016;401:557-563.

9 Petrowsky H, Gyori G, de Oliveira M, Lesurtel M, Clavien PA: Is partial-ALPPS safer than ALPPS? A singlecenter experience. Ann Surg 2015;261:e90-92.

10 Machado MA, Makdissi FF, Surjan RC, Basseres T, Schadde E: Transition from open to laparoscopic ALPPS for patients with very small FLR: the initial experience. HPB (Oxford) 2017;19:59-66.

11 Edmondson MJ, Sodergren MH, Pucher PH, Darzi A, Li J, Petrowsky H, Campos RR, Serrablo A, Jiao LR: Variations and adaptations of associated liver partition and portal vein ligation for staged hepatectomy (ALPPS): many routes to the summit. Surgery 2016;159:10581072.

12 Oldhafer KJ, Stavrou GA, van Gulik TM; Core Group: ALPPS - where do we stand, where do we go?: eight recommendations from the first International Expert Meeting. Ann Surg 2016;263:839-841.

13 Schadde E, Ardiles V, Robles-Campos R, Malago M, Machado M, Hernandez-Alejandro R, Soubrane O, Schnitzbauer AA, Raptis D, Tschuor C, Petrowsky H, De Santibanes E, Clavien PA; ALPPS Registry Group: Early survival and safety of ALPPS: first report of the International ALPPS Registry. Ann Surg 2014;260: 829-836.

14 Schadde E, Schnitzbauer AA, Tschuor C, Raptis DA, Bechstein WO, Clavien PA: Systematic review and meta-analysis of feasibility, safety, and efficacy of a novel procedure: associating liver partition and portal vein ligation for staged hepatectomy. Ann Surg Oncol 2015;22:3109-3120.

15 Li J, Girotti P, Königsrainer I, Ladurner R, Königsrainer A, Nadalin S: ALPPS in right trisectionectomy: a safe procedure to avoid postoperative liver failure? J Gastrointest Surg 2013;17:956-961.
6 Lang H, de Santibanes E, Clavien PA: Outcome of ALPPS for perihilar cholangiocarcinoma: case-control analysis including the first series from the international ALPPS registry. HPB (Oxford) 2017;19:379380.

17 Olthof PB, Coelen RJS, Wiggers JK, Groot Koerkamp B, Malago M, Hernandez-Alejandro R, Topp SA, Vivarelli M, Aldrighetti LA, Robles Campos R, Oldhafer KJ, Jarnagin WR, van Gulik TM: High mortality after ALPPS for perihilar cholangiocarcinoma: casecontrol analysis including the first series from the international ALPPS registry. HPB (Oxford) 2017;19: 381-387.

18 Vennarecci G, Grazi GL, Sperduti I, Busi Rizzi E, Felli E, Antonini M, D'Offizi G, Ettorre GM: ALPPS for primary and secondary liver tumors. Int J Surg 2016;30: 38-44.

19 Bjornsson B, Sparrelid E, Rosok B, Pomianowska E, Hasselgren K, Gasslander T, Bjornbeth BA, Isaksson B, Sandstrom P: Associating liver partition and portal vein ligation for staged hepatectomy in patients with colorectal liver metastases - intermediate oncological results. Eur J Surg Oncol 2016;42:531-537.

20 Lang SA, Loss M, Benseler V, Glockzin G, Schlitt HJ: Long-term results after in-situ split (ISS) liver resection. Langenbecks Arch Surg 2015;400:361-369.

21 Oldhafer KJ, Donati M, Jenner RM, Stang A, Stavrou GA: ALPPS for patients with colorectal liver metastases: effective liver hypertrophy, but early tumor recurrence. World J Surg 2014;38:1504-1509.

22 Ratti F, Schadde E, Masetti M, Massani M, Zanello M, Serenari M, Cipriani F, Bonariol L, Bassi N, Aldrighetti L, Jovine E: Strategies to increase the resectability of patients with colorectal liver metastases: a multi-center case-match analysis of ALPPS and conventional twostage hepatectomy. Ann Surg Oncol 2015;22:19331942. 